Chinese Journal of Organic Chemistry

\title{
串联反应合成氟磺酸联苯酚酯
}

\author{
李新民* 胡瑞陈正军胡庆红袁泽利* \\ (遵义医科大学药学院 贵州遵义 563000)
}

\begin{abstract}
摘要 发展了一个以溴代苯酚为原料通过串联反应制备氟磺酸联苯酚酯的反应体系. 该体系以钯碳为催化剂, 无水碳 酸钾为碱，乙醇水溶液为溶剂，室温下进行 Suzuki 反应后，通入硫酰氟气体，最终得到氟磺酸联苯酚酯. 反应过程中无 需分离中间体，无需加入有机膦配体及氮气保护反应，操作简便，反应条件温和，官能团底物兼容性好，产品分离收率 最高达 92.6\%; 此外, 钯碳催化剂可回收使用三次几乎不失去活性.

关键词 苯酚; 氟磺酸联苯酚酯; Suzuki 反应; 串联反应; 钯碳
\end{abstract}

\section{Preparation of Biaryl Fluorosulfates by a Tandem Process}

\author{
Li, Xinmin* Hu, Rui Chen, Zhengjun Hu, Qinghong Yuan, Zeli* \\ (College of Pharmacy, Zunyi Medical University, Zunyi, Guizhou 563000)
}

\begin{abstract}
The one-pot tandem protocol for the preparation of biaryl fluorosulfates from bromo phenols was developed. Using $\mathrm{Pd} / \mathrm{C}$ as catalyst, $\mathrm{K}_{2} \mathrm{CO}_{3}$ as base and aqueous ethanol as solvent, the Suzuki reaction was carried out at room temperature, then $\mathrm{SO}_{2} \mathrm{~F}_{2}$ gas was added to the mixture to afford biaryl fluorosulfates product. The intermediate was not isolated, and phosphine ligand and nitrogen protection were not required during the reaction, which made the protocol more convenient to operate. The one-pot protocol could tolerate a range of functional groups and provided a highest product yield up to $97.2 \%$ at room temperature. Furthermore, $\mathrm{Pd} / \mathrm{C}$ catalyst could be recycled and reused three times without significant loss of catalytic activity

Keywords phenol; baryl fluorosulfates; Suzuki reaction; one-pot reaction; $\mathrm{Pd} / \mathrm{C}$
\end{abstract}

氟磺酸酚酯 $\left(\mathrm{ArOSO}_{2} \mathrm{~F}\right)$ 作为一种新型试剂得到了广 泛关注. 2018 年 Sharpless 课题组 ${ }^{[1]}$ 报道了氟磺酸酚酯作 为亲电试剂通过 “反向药物发现” 策略探测与之相匹配 的人类蛋白质, 为人类蛋白质组的鉴定提供探针, 显示 了氟磺酸酚酯在药物开发上应用前景. 同时, 氟磺酸酚 酯基团 $\left(\mathrm{OSO}_{2} \mathrm{~F}\right)$ 可作为离去基团参与多种交叉偶联反应 及官能团化反应, 已成为丰富分子结构的重要方 法 $^{[2]}$. 开发氟磺酸酚酯的高效合成方法成为研究人员关注的 热点. 2014 年, Sharpless 及董佳家等 ${ }^{[3]}$ 报道了酚在碱性 条件下与硫酰氟气体反应, 可高收率得到氟磺酸酚酯. De Borggraeve 课题组 ${ }^{[4]}$ 报道了 $N^{\prime}, N^{\prime}$-硫酰二咪唑(SDI) 原 位生成硫酰氟, 与酚反应后制备氟磺酸酚酯, 然而反应 过程中需使用两个连接的封管, 存在较大安全风险. 2018 年, Ende 课题组 ${ }^{[5]}$ 将乙酰胺基苯双氟磺酰亚胺 (AISF)作为硫酰氟的替代物用于酚的氟磺酰酯化和仲
胺的氟磺酰胺化, 但该试剂合成步骤繁琐、价格昂贵. 2018 年, Sharpless 和董佳家 ${ }^{[6]}$ 合成了一种固体氟磺酰咪 唑盐, 该试剂同时具备良好的活性及选择性, 为氟磺酸 酯基团的引入提供了一个良好的替代方案. 虽然近年来 氟磺酸酚酯的合成报道逐渐增加, 然而高效、温和制备 氟磺酸联苯酚酯的方法未见报道.

Suzuki 反应是指过渡金属钯催化卤代芳烃与芳基 硼化物的交叉偶联反应，是构建碳一碳键的重要方法之 - ${ }^{[7]}$. 该反应具有反应条件温和、官能团兼容性好、底 物对水和空气不敏感等优点, 因此被广泛用于医药、农 药、天然产物、先进功能材料等重要精细化学品的合 成 ${ }^{[8]} .2010$ 年, 该反应的发明人 Suzuki 因在交叉偶联反 应领域的杰出贡献而获得了诺贝尔化学奖，由此可见该 反应的价值及重要性 ${ }^{[9]}$. 经历了三十多年的发展, 钯催 化的 Suzuki 反应在机理研究 ${ }^{[10]}$ 、催化剂创新 ${ }^{[11]}$ 、底物

\footnotetext{
* Corresponding authors. E-mail: lixm@zmu.edu.cn; zlyuan@zmu.edu.cn Received February 25, 2020; revised April 26, 2020; published online May 11, 2020.

Project supported by the National Natural Science Foundation of China (Nos. 81660575, 81360471), the Natural Science and Technology Foundation of Guizhou Province (No. [2018]1187), and the International Cooperation Project of Guizhou Province (No. [2020]4104).

国家自然科学基金(Nos. 81660575, 81360471)、贵州省自然科学基金(No. [2018]1187)和贵州省国际合作(No. [2020]4104)资助项目。
} 
扩展 ${ }^{[12]}$ 等方面均取得了重要进展, 然而 Suzuki 偶联反 应中常加入富电子、大位阻的膦配体以促进反应的进行. 大部分配体不仅价格昂贵且对空气敏感，需在氮气或氩 气等气体氛围下进行反应, 增加了实验操作的难度, 不 利于放大生产 ${ }^{[13]}$. 因此, 发展高效、绿色的 Suzuki 反应 体系已成为本领域的一个研究热点.

钯碳由于价格便宜、催化效率高以及易于回收, 已 成为一种具有重要工业价值的钯催化前体 ${ }^{[14]}$. 本工作 发展了一个室温下溴代苯酚串联反应合成氟磺酸联苯 酚酯的方法. 以钯碳为催化剂、无水碳酸钾为碱、乙醇 水溶液为溶剂, 不加入任何配体, 室温下进行 Suzuki 反 应, 再通入硫酰氟气体, 最终得到氟磺酸联苯酚酯.

\section{1 结果与讨论}

钯源、碱等条件对 Suzuki 反应均有较大的影响, 为 发展一个温和、高效的反应体系, 以 4-溴苯酚与苯硼酸 的 Suzuki 反应为模板, 在室温条件下考察各项条件对 Suzuki 反应的影响规律. 首先考察钯源对 Suzuki 反应的 影响规律, 结果见表 1 . 以 2 equiv. 碳酸钾作为碱时, 二 价钯与零价钯均表现出良好的催化活性, 反应 $0.5 \mathrm{~h}$ 均 得到 90\%以上的产率(表 1, Entries 1 6). 其中钯碳催化 活性最高, 可以得到 $97.2 \%$ 的产率(表 1, Entry 3), 因此 选择钯碳作为催化剂进行后续研究. 对碱的笁选发现, 无机碱如碳酸钾、碳酸钠、碳酸氢钠、碳酸铯均表现良 好的活性. 其中碳酸钾反应效果最好(表 1, Entry 3), 碳 酸钠为碱也可得到 $80.7 \%$ 的分离产率(表 1, Entry 7). Suzuki 反应常用的有机碱, 如三乙胺、二异丙基胺则

表 1 Suzuki 反应条件优化 ${ }^{a}$

Table 1 Optimization of the Suzuki reaction condition

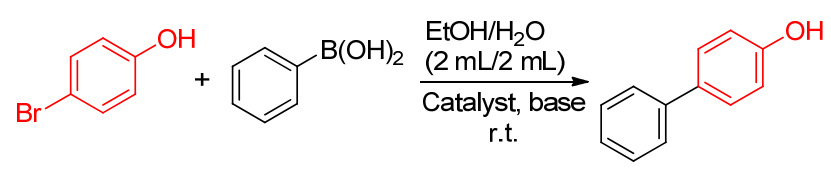

\begin{tabular}{cllc}
\hline Entry & Catalyst & \multicolumn{1}{c}{ Base } & Yield $^{b} \%$ \\
\hline 1 & $\mathrm{Pd}(\mathrm{OAc})_{2}$ & $\mathrm{~K}_{2} \mathrm{CO}_{3}$ & 95.3 \\
2 & $\mathrm{PdCl} 2$ & $\mathrm{~K}_{2} \mathrm{CO}_{3}$ & 92.4 \\
3 & $\mathrm{Pd} / \mathrm{C}$ & $\mathrm{K}_{2} \mathrm{CO}_{3}$ & 97.2 \\
4 & $\mathrm{Pd} / \mathrm{Al}_{2} \mathrm{O}_{3}$ & $\mathrm{~K}_{2} \mathrm{CO}_{3}$ & 90.7 \\
5 & $\mathrm{Pd} / \mathrm{BaSO}_{4}$ & $\mathrm{~K}_{2} \mathrm{CO}_{3}$ & 91.9 \\
6 & $\mathrm{Pd} / \mathrm{CaCO}_{3}$ & $\mathrm{~K}_{2} \mathrm{CO}_{3}$ & 94.1 \\
7 & $\mathrm{Pd} / \mathrm{C}$ & $\mathrm{Na}_{2} \mathrm{CO}_{3}$ & 80.7 \\
8 & $\mathrm{Pd} / \mathrm{C}$ & $\mathrm{NaHCO}_{3}$ & 78.9 \\
9 & $\mathrm{Pd} / \mathrm{C}$ & $\mathrm{Cs}_{2} \mathrm{CO}_{3}$ & 79.3 \\
10 & $\mathrm{Pd} / \mathrm{C}$ & $\mathrm{Et}_{3} \mathrm{~N}$ & 51.5 \\
11 & $\mathrm{Pd} / \mathrm{C}$ & $(i-\mathrm{Pr})_{2} \mathrm{NH}$ & 62.7 \\
\hline
\end{tabular}

${ }^{a}$ Reaction conditions: 4-bromonitrophenol $(0.5 \mathrm{mmol})$, phenylboronic acid $(0.6 \mathrm{mmol})$, base $(1.0 \mathrm{mmol}), \mathrm{Pd}$ catalyst $(0.5 \mathrm{~mol} \%), \mathrm{EtOH} / \mathrm{H}_{2} \mathrm{O}(2 \mathrm{~mL} / 2$ $\mathrm{mL}), 25{ }^{\circ} \mathrm{C}$, in air, $30 \mathrm{~min} .{ }^{b}$ Isolated yield.
表现出较差的反应活性，分别得到 $51.5 \%$ 及 $62.7 \%$ 的产 率(表 1, Entries 21, 22). 因此确定 Suzuki 偶联反应的最 佳催化剂为钯碳, 碱为碳酸钾.

第二步的条件优化中以 4-羟基联苯为原料, 室温下 通入硫酰氟气体，考察不同碱对反应的影响规律. 从表 2 中可以发现, 有机碱对反应的促进效果优于无机碱. 当使用 5 equiv.碳酸钾为碱时，可以得到 $71.2 \%$ 的产率 (表 2, Entry 1). 碳酸钠、碳酸铯作为碱分别可提供 $78.0 \%$ 及 $81.6 \%$ 的产率(表 2, Entries 2, 3). 有机碱三乙胺及二 异丙胺均表现出良好的活性, 其中使用三乙胺为碱可得 到 92.7\%的产率(表 2, Entry 5). 降低三乙胺的量对反应 产率影响较大. 当使用 2 equiv.三乙胺时, 反应 $0.5 \mathrm{~h}$ 仅 得到 $42.1 \%$ 的产率(表 2, Entry 8). 综合考虑, 选择 5 equiv.三乙胺为碱进行后续研究.

表 2 第二步反应条件优化 ${ }^{a}$

Table 2 Optimization of the second step reaction condition

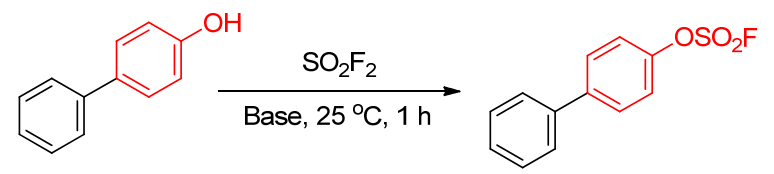

\begin{tabular}{cccc}
\hline Entry & Base & Base/equiv. & Yield $^{b} / \%$ \\
\hline 1 & $\mathrm{~K}_{2} \mathrm{CO}_{3}$ & 5.0 & 71.2 \\
2 & $\mathrm{Na}_{2} \mathrm{CO}_{3}$ & 5.0 & 78.0 \\
3 & $\mathrm{Cs}_{2} \mathrm{CO}_{3}$ & 5.0 & 81.6 \\
4 & $\left(i-\mathrm{Pr}_{2} \mathrm{NH}\right.$ & 5.0 & 85.1 \\
5 & $\mathrm{Et}_{3} \mathrm{~N}$ & 5.0 & 92.7 \\
6 & $\mathrm{Et}_{3} \mathrm{~N}$ & 4.0 & 84.6 \\
7 & $\mathrm{Et}_{3} \mathrm{~N}$ & 3.0 & 71.7 \\
8 & $\mathrm{Et}_{3} \mathrm{~N}$ & 2.0 & 42.1 \\
\hline
\end{tabular}

${ }^{a}$ Reaction conditions: 4-hydroxybiphenyl $(0.5 \mathrm{mmol})$, base (2.5 mmol), $\mathrm{SO}_{2} \mathrm{~F}_{2}$ gas, $\mathrm{EtOH} / \mathrm{H}_{2} \mathrm{O}(2 \mathrm{~mL} / 2 \mathrm{~mL}), 25{ }^{\circ} \mathrm{C}$, in air, $30 \mathrm{~min} .{ }^{b}$ Isolated yield

最优反应条件下, 即 $1 \mathrm{~mol} \%$ 钯碳催化剂、2 equiv. 碳酸钾为碱、乙醇一水溶液为溶剂、 $25{ }^{\circ} \mathrm{C}$ 下搅拌反应, 原 料完全反应后, 通入磺酰氟气体, 并加入 5 equiv.三乙 胺, $25{ }^{\circ} \mathrm{C}$ 下继续反应，考察该体系的底物适用范围，实 验结果列于表 3. 首先考察底物中取代基电子效应对反 应活性的影响，以 4-溴苯酚及含吸电子基团的 4-氧基苯 硼酸为底物时, 最终产率较低, 仅为 $18.1 \%$ (表 3,1 b). 主 要因为氰基的吸电性使硼酸活性变低，导致第一步偶联 反应进行困难，后处理以后发现大量 4-溴苯酚原料剩 余. 以 4 -氟苯硼酸或 4 -氯苯硼酸为底物时, 得到中等的 产率(表 3, 1c, 1d). 然而含吸电性较强的甲酰基和乙酰 基硼酸为底物时，几乎没有产品生成，原料 4-澳苯酚大 量剩余(表 $3,1 \mathrm{e}, \mathbf{1 f}$ ). 当使用含给电子基团的芳基硼酸 为底物时, 反应均能顺利进行并得到良好的产率(表 3 , $1 g \sim 1 n)$. 如以 4-甲氧基苯硼酸为底物, “一锅”反应 $6 \mathrm{~h}$ 
后可得到 92.6\%的产率(表 3, 1g). 以 4-叔丁基苯硼酸为 底物, “一锅”反应 $6 \mathrm{~h}$ 可得到 $84.9 \%$ 的产率(表 3,1n). 通 过以上数据的分析, 作者认为第一步偶联反应为速率控 制步, 使用含电子基团的芳基硼酸有利于反应的进行. 进一步对 $N$-杂环硼酸为底物的反应进行了考察, 分别 得到 $25.6 \%$ 和 $53.0 \%$ 的产率 (表 3, 1o, 1p). 然而, 以噻吩 嗍酸为底物时, 没有任何产品生成, 原因是噻吩硼酸在 室温下不与 4-澳苯酚发生偶联反应(表 3, 1r). 间溴苯酚 与不同芳基硼酸的反应结果表明, 间澳苯酚与含有给电 子基团的芳基硼酸均可较好地进行反应, 得到 $80 \%$ 以上 的产率(表 3, 1s $\sim 1 \mathbf{u}$ ). 然而, 邻溴苯酚由于受到空间位
阻的影响，与芳基硼酸反应仅得到中等产率(表 $3,1 \mathrm{v} \sim$ $1 \mathbf{x}$ ). 当使用 $N$-杂环溴代酚或䒺酚为底物时, 反应 $12 \mathrm{~h}$ 时没有产物生成(表 3, 1y $\sim 1 \mathbf{z}$ ). 从工业化和环境保护的 角度来看, 钯催化剂的回收和重复使用具有重要价值. 因此，作者对反应体系中非均相催化剂钯碳的重复使用 能力做了研究. 循环使用实验以 4-澳苯酚与 4-甲氧基苯 硼酸的偶联反应为模型反应, $1 \mathrm{~mol} \%$ 的钯碳化剂, 在室 温和空气条件下反应 $6 \mathrm{~h}$. 在每次反应结束以后, 经过 简单的过滤，并依次用乙酸乙酯、水洗涤，自然晾干后， 直接用于下一次反应. 实验结果如表 4 所示, 在该非均 相催化体系中，钯碳催化剂使用的前三次，活性基本保

表 3 联芳氟磺酸酯的合成 ${ }^{a}$

Table 3 Preparation of biaryl fluorosulfates

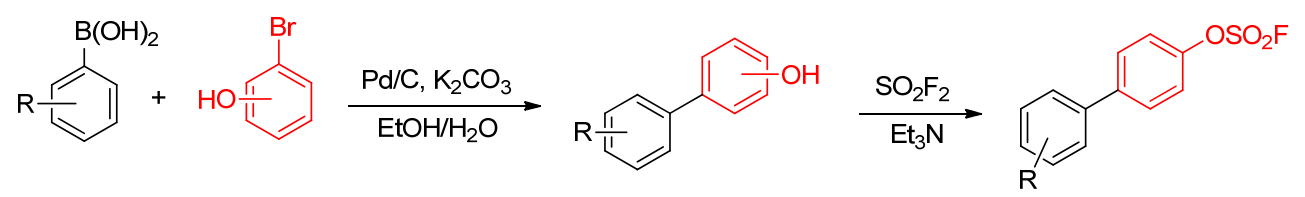

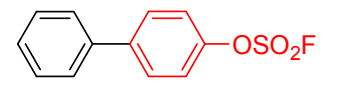

1a, $1 \mathrm{~h}, 90.1 \%$
$\mathrm{NC}$

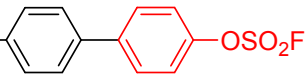

1b, $5 \mathrm{~h}, 18.1 \%$

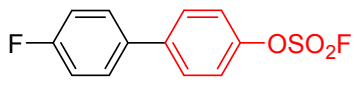

1c, $5 \mathrm{~h}, 70.2 \%$

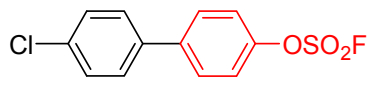

1d, 6 h, $63.9 \%$

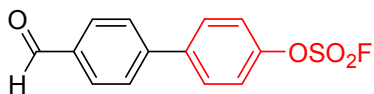

1e, $6 \mathrm{~h}$, trace

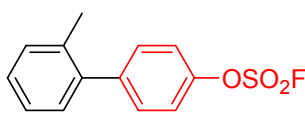

1i, $6 \mathrm{~h}$, trace

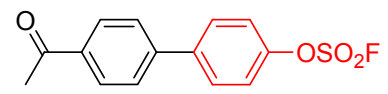

1f, $6 \mathrm{~h}$, trace

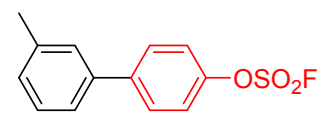

1j, $6 \mathrm{~h}, 82.6 \%$

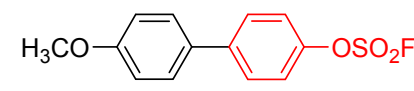

1g, 6 h, $92.6 \%$

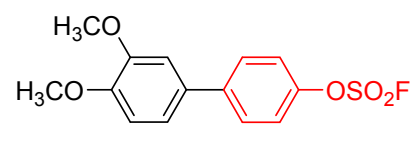

1h, $14 \mathrm{~h}, 81.3 \%$

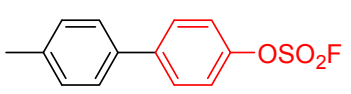

$1 \mathrm{k}, 6 \mathrm{~h}, 93.6 \%$

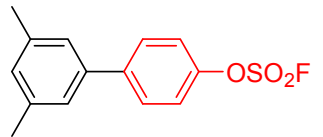

1I, 6 h, $78.0 \%$

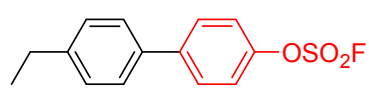

1m, 7 h, $85.2 \%$

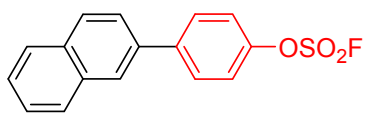

1q, 8 h, $48.9 \%$

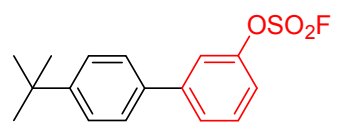

$1 \mathrm{u}, 8 \mathrm{~h}, 83.4 \%$

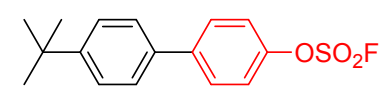

1n, 6 h, $84.9 \%$

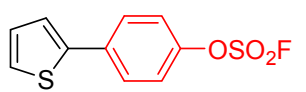

1r, 8 h, 0

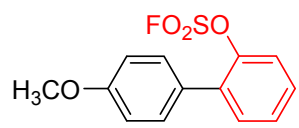

1v, 8 h, $57.8 \%$

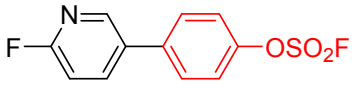

1o, $12 \mathrm{~h}, 25.6 \%$

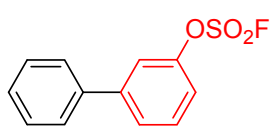

1s, 4 h, $92.9 \%$

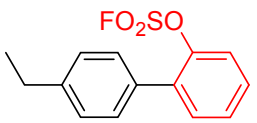

1w, 8 h, $60.2 \%$

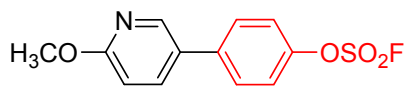

$1 \mathrm{p}, 12 \mathrm{~h}, 53.0 \%$

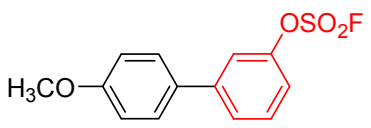

1t, 6 h, $81.6 \%$

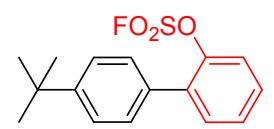

1x, 8 h, $44.6 \%$<smiles>Cc1ccc(-c2ccc(OS(=O)(=O)F)cn2)cc1</smiles>

$1 y, 12 \mathrm{~h}, 0^{b}$<smiles>[Y20]#[R6]</smiles>

${ }^{a}$ Reaction conditions: first step: bromo phenol $(0.5 \mathrm{mmol})$, aryboronic acid $(0.6 \mathrm{mmol}), \mathrm{K}_{2} \mathrm{CO}_{3}(1.0 \mathrm{mmol}), \mathrm{Pd} / \mathrm{C}(0.5 \mathrm{~mol} \%), \mathrm{EtOH} / \mathrm{H}_{2} \mathrm{O}(2 \mathrm{~mL} / 2 \mathrm{~mL}), 25{ }^{\circ} \mathrm{C}$, in air; after $0.5 \sim 3.0 \mathrm{~h}, \mathrm{Et}_{3} \mathrm{~N}(2.5 \mathrm{mmol}), \mathrm{SO}_{2} \mathrm{~F}_{2}$ gas were added, $25{ }^{\circ} \mathrm{C}, 0.5 \sim 5 \mathrm{~h}$, isolated yield. ${ }^{b} 80{ }^{\circ} \mathrm{C}$ for the first step. 
持不变, 第四次时收率降到 68.4\%. 作者推测主要原因 是每次催化剂的回收均有损失, 钯碳使用量的降低导致 产率下降. 由以上数据可知, 在此催化体系中钯碳催化 剂有着良好的循环使用能力.

表 4 钯碳的循环回收 ${ }^{a}$

Table 4 Recycling of $\mathrm{Pd} / \mathrm{C}$
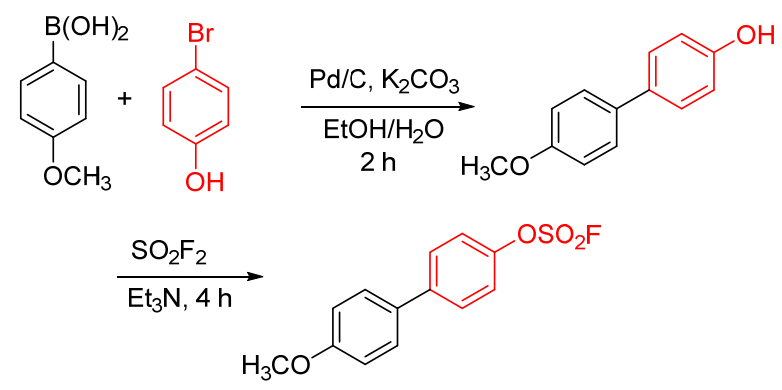

\begin{tabular}{ccc}
\hline Entry & Run & Yield $^{b} \%$ \\
\hline 1 & 1 & 92.6 \\
2 & 2 & 89.1 \\
3 & 3 & 84.2 \\
4 & 4 & 68.4 \\
5 & 5 & 46.1 \\
\hline
\end{tabular}

${ }^{a}$ Reaction conditions: first step: 4-bromophenol (2 mmol), 4-methoxylpehnylboronic acid (2.4 mmol), $\mathrm{K}_{2} \mathrm{CO}_{3}(40 \mathrm{mmol}), \mathrm{Pd} / \mathrm{C}(1.0 \mathrm{~mol} \%), \mathrm{EtOH} / \mathrm{H}_{2} \mathrm{O}(5$ $\mathrm{mL} / 5 \mathrm{~mL}), 25{ }^{\circ} \mathrm{C}$, in air; after $3.0 \mathrm{~h}, \mathrm{Et}_{3} \mathrm{~N}(10.0 \mathrm{mmol}), \mathrm{SO}_{2} \mathrm{~F}_{2}$ gas were added, $25{ }^{\circ} \mathrm{C}, 3.0$ h. ${ }^{b}$ Isolated yield

\section{2 结论}

发展了一种以溴代苯酚为原料 “一锅” 制备氟磺酸 联苯酚酯的方法. 首先钯碳催化下室温 Suzuki 偶联反应 制备联苯酚, 无需分离中间体，补加三乙胺及通入硫酰 氟气体后，最终制备得到氟磺酸联苯酚酯. 该体系反应 条件温和，底物兼容性好，产物最高收率可达 $92.6 \%$. 此外, 钯碳可通过简单过滤, 可回收使用三次, 具有良 好的工业应用的潜力.

\section{3 实验部分}

\section{1 仪器与试剂}

卤代苯酚及芳基硼酸购自上海萨恩化学技术有限 公司, 硫酰氟气体购自武汉纽瑞德特种气体有限公司; 其他试剂均为国产分析纯试剂, 未经进一步处理, 直接 使用; 柱层析硅胶(试剂级, 200 300 目)购自青岛海洋 化工有限公司，直接使用. Bruker ADVANCEII 400 型核 磁共振仪, TMS 为内标, 氝代氯仿为溶剂.

\section{2 实验方法}

\subsection{1串联反应制备氟磺酸联苯酚酯的一般步骤}

在空气中, 依次将溴代苯酚 $(0.5 \mathrm{mmol})$ 、芳基硼酸 $(0.6 \mathrm{mmol})$ 、无水碳酸钾 $(1 \mathrm{mmol}) 、 10 \%$ 钯碳 $(0.5 \mathrm{~mol} \%)$ 、
$50 \%$ 乙醇水溶液( $4 \mathrm{~mL}$ )加入到 $10 \mathrm{~mL}$ 长颈瓶中, 室温反 应 $0.5 \sim 3.0 \mathrm{~h}$. 薄层色谱板检测溴代酚反应完后, 加入 碱(2.5 mmol), 再用气球导入硫酰氟气体，继续反应 $0.5 \sim 5.0 \mathrm{~h}$. 薄层色谱板检测反应结束后, 加入饱和氯化 钠和乙酸乙酯萃取，萃取后有机层减压浓缩，粗产品经 柱层析获得目标产物, 洗脱剂为石油醚与乙酸乙酯.

\section{2 .2 钯碳回收实验}

在空气中, 依次将 4-溴苯酚(2 mmol)、4-甲氧基苯 喼酸 $(2.4 \mathrm{mmol})$ 、无水碳酸钾 $(4 \mathrm{mmol}) 、 10 \%$ 钯碳 $(0.5$ $\mathrm{mol} \%$ )和 $50 \%$ 乙醇水溶液 $(10 \mathrm{~mL})$ 加入 $50 \mathrm{~mL}$ 长颈瓶中, 室温反应 $3.0 \mathrm{~h}$, 然后加入三乙胺 $(10 \mathrm{mmol})$, 再用气球 通入硫酰氟气体，继续反应 $3 \mathrm{~h}$. 反应结束后，将反应液 用滤纸直接过滤, 依次用乙酸乙酯 $(20 \mathrm{~mL})$ 、去离子水 $(20 \mathrm{~mL})$ 洗涤滤饼，然后将滤饼自然晾干即回收钯碳，直 接用于下一次反应. 其滤液加入饱和 $\mathrm{NaCl}$ 溶液, 乙酸 乙酯萃取, 合并有机相, 使用旋转蒸发仪浓缩得到粗产 品, 经柱层析分离得到分析纯的目标产物. 在下次实验 时，将晾干的钯碳继续投入到模板反应中，重复进行反 应.

\subsection{3 联芳环氟磺酰酯表征数据}

氟磺酸联苯酚酯 $(\mathbf{1 a})^{[15]}$ : 白色固体，m.p. 92.0 93.6 ${ }^{\circ} \mathrm{C}$ (文献值 ${ }^{[16]} 94.3 \sim 95.4{ }^{\circ} \mathrm{C}$ ); ${ }^{1} \mathrm{H}$ NMR (400 MHz, $\left.\mathrm{CDCl}_{3}\right) \delta: 7.67(\mathrm{~d}, J=8.5 \mathrm{~Hz}, 2 \mathrm{H}), 7.56(\mathrm{~d}, J=7.7 \mathrm{~Hz}$, $2 \mathrm{H}), 7.48(\mathrm{t}, J=7.5 \mathrm{~Hz}, 2 \mathrm{H}), 7.44 \sim 7.32(\mathrm{~m}, 3 \mathrm{H}) ;{ }^{13} \mathrm{C}$ NMR (101 MHz, $\left.\mathrm{CDCl}_{3}\right) \delta: 149.5,142.1,139.3,129.1$, 128.2, 127.3, 121.3; IR (KBr) v: 3430, 2912, 1903, 1472, $1442,1208,1174,1045,896,625,553 \mathrm{~cm}^{-1}$.

氟磺酸 4-氰基联苯酚酯 $(\mathbf{1 b})^{[2 \mathrm{~b}]}$ : 白色固体, m.p. $79.0 \sim 80.5{ }^{\circ} \mathrm{C} ;{ }^{1} \mathrm{H}$ NMR $\left(400 \mathrm{MHz}, \mathrm{CDCl}_{3}\right) \delta: 7.69(\mathrm{~d}$, $J=8.1 \mathrm{~Hz}, 2 \mathrm{H}), 7.60 \sim 7.53(\mathrm{~m}, J=7.6 \mathrm{~Hz}, 4 \mathrm{H}), 7.40(\mathrm{~d}$, $J=8.5 \mathrm{~Hz}, 2 \mathrm{H}) ;{ }^{13} \mathrm{C}$ NMR $\left(101 \mathrm{MHz}, \mathrm{CDCl}_{3}\right) \delta: 150.0$, $143.5,139.8,133.5,132.7,129.2,127.8,121.6,118.4$, 111.8; IR (KBr) v: 3402, 3066, 2224, 1238, 1607, 1491, 1442 1235, 1143 920, 825, 918, 593, $544 \mathrm{~cm}^{-1}$. HRMS (ESI) calcd for $\mathrm{C}_{13} \mathrm{H}_{8} \mathrm{FNO}_{3} \mathrm{SNa}[\mathrm{M}+\mathrm{Na}]^{+}$300.0107, found 300.0098 .

氟磺酸 4-氟联苯酚酯 $(1 \mathrm{c})^{[4]}$ : 白色固体, m.p. 60.0 $61.5{ }^{\circ} \mathrm{C} ;{ }^{1} \mathrm{H}$ NMR $\left(400 \mathrm{MHz}, \mathrm{CDCl}_{3}\right) \delta: 7.48$ (d, $J=8.2$ $\mathrm{Hz}, 3 \mathrm{H}), 7.40 \sim 7.37(\mathrm{~m}, 2 \mathrm{H}), 7.28(\mathrm{~d}, 2 \mathrm{H}), 7.00$ (s, 2H). ${ }^{13} \mathrm{C}$ NMR $\left(101 \mathrm{MHz}, \mathrm{CDCl}_{3}\right) \delta: 163.0,160.5,148.2,139.8$, 132.4, 127.8, 120.2, 114.9.

氟磺酸 4-氯联苯酚酯 $(1 d)$ : 白色固体，m.p. 63.0 $65.0{ }^{\circ} \mathrm{C} ;{ }^{1} \mathrm{H}$ NMR (400 MHz, $\mathrm{CDCl}_{3}$ ) $\delta: 7.63$ (d, $J=7.9$ $\mathrm{Hz}, 2 \mathrm{H}), 7.49$ (d, $J=7.8 \mathrm{~Hz}, 2 \mathrm{H}), 7.46 \sim 7.39(\mathrm{~m}, 4 \mathrm{H}) ;{ }^{13} \mathrm{C}$ NMR (101 MHz, $\left.\mathrm{CDCl}_{3}\right) \delta: 149.8,140.7,137.6,134.3$, 
129.18, 128.88, 128.43, 121.34; IR (KBr) v: 3431, 2924, $1451,1141,913,822,918,585,542 \mathrm{~cm}^{-1}$. HRMS (ESI) calcd for $\mathrm{C}_{13} \mathrm{H}_{8} \mathrm{ClNO}_{3} \mathrm{SNa}[\mathrm{M}+\mathrm{Na}]^{+}$308.9760, found 308.9758 .

氟磺酸 4-甲氧基联苯酚酯 $(\mathbf{1 g})^{[17]}$ : 白色固体, m.p. $78.6 \sim 80.0{ }^{\circ} \mathrm{C} ;{ }^{1} \mathrm{H}$ NMR (400 MHz, $\left.\mathrm{CDCl}_{3}\right) \delta: 7.54(\mathrm{~d}$, $J=7.4 \mathrm{~Hz}, 2 \mathrm{H}), 7.41$ (d, $J=7.4 \mathrm{~Hz}, 2 \mathrm{H}), 7.30$ (d, $J=8.2$ $\mathrm{Hz}, 2 \mathrm{H}), 6.91$ (d, $J=7.4 \mathrm{~Hz}, 2 \mathrm{H}), 3.78$ (s, 3H); ${ }^{13} \mathrm{C} \mathrm{NMR}$ $\left(101 \mathrm{MHz}, \mathrm{CDCl}_{3}\right) \delta: 159.5,148.7,141.4,131.5,128.3$, 128.1, 126.8, 120.9, 114.2, 55.2; IR (KBr) v: 3430, 2964, $1607,1493,1453,1235,1200,1151,924,822,602,541$ $\mathrm{cm}^{-1}$. HRMS (ESI) calcd for $\mathrm{C}_{13} \mathrm{H}_{11} \mathrm{FO}_{3} \mathrm{SNa}[\mathrm{M}+\mathrm{Na}]^{+}$ 305.02543 , found 305.02432 .

氟磺酸 3,4-二甲氧基联苯酚酯 $(\mathbf{1 h})^{[17]}$ : 白色固体, m.p. 97.0 99.5 ${ }^{\circ} \mathrm{C} ;{ }^{1} \mathrm{H}$ NMR $\left(400 \mathrm{MHz}, \mathrm{CDCl}_{3}\right) \delta$ : 7.62 (d, $J=6.4 \mathrm{~Hz}, 2 \mathrm{H}), 7.38$ (d, $J=6.7 \mathrm{~Hz}, 2 \mathrm{H}), 7.08$ (d, $J=$ $19.1 \mathrm{~Hz}, 2 \mathrm{H}), 6.96(\mathrm{~s}, 1 \mathrm{H}), 3.93(\mathrm{~s}, 6 \mathrm{H}) ;{ }^{13} \mathrm{C}$ NMR (101 $\left.\mathrm{MHz}, \mathrm{CDCl}_{3}\right) \delta: 149.3,149.2,149.0,141.8,132.1,128.6$, 121.1, 119.6, 111.5, 110.3, 55.9; IR (KBr) v: 3435, 2960, 2924, 2852, 1494, 1453, 1447, 1254, 1024, 918, 813, 764, $614,512 \mathrm{~cm}^{-1}$. HRMS (ESI) calcd for $\mathrm{C}_{14} \mathrm{H}_{13} \mathrm{FO}_{5} \mathrm{SNa}[\mathrm{M}$ $+\mathrm{Na}]^{+}$335.0365, found 335.0359.

氟磺酸 3-甲基联苯酚酯(1jj $)^{[17]}$ : 无色液体. ${ }^{1} \mathrm{H}$ NMR $\left(400 \mathrm{MHz}, \mathrm{CDCl}_{3}\right) \delta: 7.50(\mathrm{~d}, J=8.6 \mathrm{~Hz}, 2 \mathrm{H}), 7.26$ (s, 1H), $7.25 \sim 7.20(\mathrm{~m}, 4 \mathrm{H}), 7.09(\mathrm{~s}, 1 \mathrm{H}), 2.30(\mathrm{~s}, 3 \mathrm{H}) ;{ }^{13} \mathrm{C}$ NMR (101 MHz, $\left.\mathrm{CDCl}_{3}\right) \delta: 149.3,142.1,139.2,138.7$, 129.0, 128.9, 128.89, 128.0, 124.3, 121.1, 21.5; IR (KBr) v: 3362, 2974, 2894, 1453, 1089, 1049, 918, 881, 579 $\mathrm{cm}^{-1}$. HRMS (ESI) calcd for $\mathrm{C}_{13} \mathrm{H}_{12} \mathrm{FO}_{5} \mathrm{~S}[\mathrm{M}+\mathrm{H}]^{+}$ 267.0491, found 267.0474.

氟磺酸 4-甲基联苯酚酯 $(\mathbf{1 k})^{[17]}$ : 白色固体, m.p. $64.0 \sim 65.0{ }^{\circ} \mathrm{C} ;{ }^{1} \mathrm{H}$ NMR $\left(400 \mathrm{MHz}, \mathrm{CDCl}_{3}\right) \delta: 7.55$ (d, $J=8.7 \mathrm{~Hz}, 2 \mathrm{H}), 7.36$ (d, $J=8.0 \mathrm{~Hz}, 2 \mathrm{H}), 7.30$ (d, $J=8.5$ $\mathrm{Hz}, 2 \mathrm{H}), 7.18$ (d, $J=7.9 \mathrm{~Hz}, 2 \mathrm{H}), 2.32$ (s, 3H). ${ }^{13} \mathrm{C} \mathrm{NMR}$ $\left(101 \mathrm{MHz}, \mathrm{CDCl}_{3}\right) \delta: 149.1,141.8,138.0,136.3,129.6$, 128.7, 127.0, 121.0, 21.1; IR (KBr) v: 3435, 2925, 1913, 1492, 1456, 1237, 1184, 1145, 912, 809, 602, $555 \mathrm{~cm}^{-1}$. HRMS (ESI) calcd for $\mathrm{C}_{13} \mathrm{H}_{11} \mathrm{FO}_{5} \mathrm{SNa}\left[\mathrm{M}+\mathrm{Na}{ }^{+}\right.$ 289.0311, found 289.0293.

氟磺酸 3,5-二甲基联苯酚酯(11): 白色固体，m.p. 97.2 98.5 ${ }^{\circ} \mathrm{C} ;{ }^{1} \mathrm{H}$ NMR (400 MHz, $\mathrm{CDCl}_{3}$ ) $\delta: 7.48$ (d, $J=8.4 \mathrm{~Hz}, 2 \mathrm{H}), 7.22(\mathrm{~d}, J=8.7 \mathrm{~Hz}, 2 \mathrm{H}), 7.02$ (s, 2H), 6.91 $(\mathrm{s}, 1 \mathrm{H}), 2.25(\mathrm{~s}, 6 \mathrm{H}) ;{ }^{13} \mathrm{C} \mathrm{NMR}\left(101 \mathrm{MHz}, \mathrm{CDCl}_{3}\right) \delta$ : $148.3,141.3,138.3,137.6,128.8,128.0,124.2,120.1$, 20.4; IR (KBr) v: 3434, 2961, 1904, 1600, 1523, 1494,
1445, 1234, 1141, 1024, 918, 843, 813, 614, 571, 516 $\mathrm{cm}^{-1}$. HRMS (ESI) calcd for $\mathrm{C}_{14} \mathrm{H}_{14} \mathrm{FO}_{5} \mathrm{~S}[\mathrm{M}+\mathrm{H}]^{+}$ 281.0648, found 281.0642 .

氟磺酸 4-乙基联苯酚酯 (1m): 白色固体, m.p. $58.1 \sim 58.5{ }^{\circ} \mathrm{C} ;{ }^{1} \mathrm{H}$ NMR $\left(400 \mathrm{MHz}, \mathrm{CDCl}_{3}\right) \delta: 7.65(\mathrm{~d}$, $J=7.9 \mathrm{~Hz}, 2 \mathrm{H}), 7.48$ (d, $J=7.4 \mathrm{~Hz}, 2 \mathrm{H}), 7.39$ (d, $J=8.0$ $\mathrm{Hz}, 2 \mathrm{H}), 7.30$ (d, $J=7.4 \mathrm{~Hz}, 2 \mathrm{H}), 2.71$ (q, $J=7.3 \mathrm{~Hz}, 2 \mathrm{H}$ ), $1.28(\mathrm{t}, J=7.5 \mathrm{~Hz}, 3 \mathrm{H}) ;{ }^{13} \mathrm{C} \mathrm{NMR}\left(101 \mathrm{MHz}, \mathrm{CDCl}_{3}\right) \delta$ : $149.2,144.5,142.0,136.7,128.9,128.6,127.2,121.2$, 28.6, 15.6; IR (KBr) v: 3432, 2791, 1903, 1456, 1235, 1185, 1147, 914, 825, 601, 542, $508 \mathrm{~cm}^{-1}$. HRMS (ESI-TOF) calcd for $\mathrm{C}_{14} \mathrm{H}_{13} \mathrm{FO}_{3} \mathrm{SNa}[\mathrm{M}+\mathrm{Na}]^{+}$303.0467, found 303.0450 .

氟磺酸 4-叔丁基联苯酚酯(1n): 白色固体, m.p. $75.5 \sim 76.0{ }^{\circ} \mathrm{C} ;{ }^{1} \mathrm{H}$ NMR $\left(400 \mathrm{MHz}, \mathrm{CDCl}_{3}\right) \delta: 7.66(\mathrm{~d}$, $J=8.0 \mathrm{~Hz}, 2 \mathrm{H}), 7.50$ (s, 4H), 7.39 (d, $J=8.0 \mathrm{~Hz}, 2 \mathrm{H}), 1.37$ $(\mathrm{s}, 9 \mathrm{H}) ;{ }^{13} \mathrm{C}$ NMR $\left(101 \mathrm{MHz}, \mathrm{CDCl}_{3}\right) \delta: 151.2,149.1$, $141.8,136.3,128.8,126.8,125.9,121.1,34.6,31.3$; IR (KBr) v: 3435, 2967, 2871, 1923, 1493, 1452, 1235, 1146, 912, 829, 592, $521 \mathrm{~cm}^{-1}$. HRMS (ESI-TOF) calcd for $\mathrm{C}_{16} \mathrm{H}_{18} \mathrm{FO}_{3} \mathrm{~S}[\mathrm{M}+\mathrm{H}]^{+}$331.0780, found 331. 0763.

氟磺酸 4-氟-3-吡啶基联苯酚酯(10) 白色固体, m.p. $105.8 \sim 107.0{ }^{\circ} \mathrm{C} ;{ }^{1} \mathrm{H}$ NMR (400 MHz, $\mathrm{CDCl}_{3}$ ) $\delta: 8.41(\mathrm{~s}$, 1H), 7.96 (t, $J=7.8 \mathrm{~Hz}, 1 \mathrm{H}), 7.64$ (d, $J=7.9 \mathrm{~Hz}, 2 \mathrm{H}), 7.45$ $(\mathrm{d}, J=7.9 \mathrm{~Hz}, 2 \mathrm{H}), 7.05(\mathrm{~d}, J=8.4 \mathrm{~Hz}, 1 \mathrm{H}) ;{ }^{13} \mathrm{C} \mathrm{NMR}$ $\left(101 \mathrm{MHz}, \mathrm{CDCl}_{3}\right) \delta: 164.8,162.4,150.0,146.1,139.9$, $137.7,129.2$, 121.9, 109.9; IR (KBr) v: 3431, 3072, 2926, 2871, 1915, 1587, 1445, 1234, 1195, 1141, 922, 831, 605, $567,542 \mathrm{~cm}^{-1}$. HRMS (ESI-TOF) calcd for $\mathrm{C}_{11} \mathrm{H}_{8} \mathrm{~F}_{2} \mathrm{NO}_{3} \mathrm{~S}$ $[\mathrm{M}+\mathrm{H}]^{+}$272.0193, found 272.0171.

氟磺酸 4-甲氧基-3-吡啶基联苯酚酯(1p) 白色固体, m.p. $75.3 \sim 76.3{ }^{\circ} \mathrm{C} ;{ }^{1} \mathrm{H}$ NMR (400 $\mathrm{MHz}, \mathrm{CDCl}_{3}$ ) $\delta: 8.37$ (s, 1H), $7.76(\mathrm{~d}, J=8.4 \mathrm{~Hz}, 1 \mathrm{H}), 7.61(\mathrm{~d}, J=8.1 \mathrm{~Hz}, 2 \mathrm{H})$, 7.42 (d, $J=8.1 \mathrm{~Hz}, 2 \mathrm{H}), 6.85$ (d, $J=8.5 \mathrm{~Hz}, 1 \mathrm{H}), 3.99$ (s, $3 \mathrm{H}) .{ }^{13} \mathrm{C} \mathrm{NMR}\left(101 \mathrm{MHz}, \mathrm{CDCl}_{3}\right) \delta: 164.0,149.2,145.1$, 138.7, 137.3, 128.5, 121.5, 111.1, 53.6; IR (KBr) v: 3439, 3009, 2941, 1905, 1610, 1483, 1437, 1291, 1234, 1190, 1140, 1017, 949, 827, 614, $580 \mathrm{~cm}^{-1}$. HRMS (ESI-TOF) calcd for $\mathrm{C}_{12} \mathrm{H}_{11} \mathrm{FNO}_{4} \mathrm{~S}[\mathrm{M}+\mathrm{H}]^{+}$284.0393, found 284 . 0374.

氟磺酸 4-(2-菜基)苯酚酯 (1q): 白色固体, m.p. $113.0 \sim 114.0{ }^{\circ} \mathrm{C} ;{ }^{1} \mathrm{H}$ NMR (400 MHz, $\mathrm{CDCl}_{3}$ ) $\delta: 8.00(\mathrm{~s}$, $1 \mathrm{H}), 7.98 \sim 7.87(\mathrm{~m}, 3 \mathrm{H}), 7.78(\mathrm{~d}, J=8.0 \mathrm{~Hz}, 2 \mathrm{H}), 7.68(\mathrm{~d}$, $J=8.5 \mathrm{~Hz}, 1 \mathrm{H}), 7.53(\mathrm{~d}, J=4.1 \mathrm{~Hz}, 2 \mathrm{H}), 7.44$ (d, $J=8.0$ $\mathrm{Hz}, 2 \mathrm{H}) ;{ }^{13} \mathrm{C}$ NMR (101 MHz, $\left.\mathrm{CDCl}_{3}\right) \delta: 149.3,142.1$, 
$136.7,133.6,132.9,129.4,128.9,128.4,127.6,126.8$, 126.6, 126.4, 125.1, 121.2; IR (KBr) v: 3432, 3047, 2923, 1499, 1441, 1235, 953, 935, 819, 750, 599, $566 \mathrm{~cm}^{-1}$. HRMS (ESI-TOF) calcd for $\mathrm{C}_{16} \mathrm{H}_{12} \mathrm{FNO}_{3} \mathrm{~S}[\mathrm{M}+\mathrm{H}]^{+}$ 303.0491, found 303.0469.

氟磺酸 3-(苯基)苯酚酯(1s) ${ }^{[15]}$ : 白色固体, m.p. 49.1 50.5 ${ }^{\circ} \mathrm{C} ;{ }^{1} \mathrm{H}$ NMR (400 MHz, $\left.\mathrm{CDCl}_{3}\right) \delta$ : 7.61 (d, $J=7.7 \mathrm{~Hz}, 2 \mathrm{H}), 7.53(\mathrm{dd}, J=14.0,8.4 \mathrm{~Hz}, 4 \mathrm{H}), 7.45$ (t, $J=7.3 \mathrm{~Hz}, 2 \mathrm{H}), 7.40(\mathrm{~d}, J=6.8 \mathrm{~Hz}, 1 \mathrm{H}), 7.29$ (d, $J=8.1$ $\mathrm{Hz}, 1 \mathrm{H}) .{ }^{13} \mathrm{C} \mathrm{NMR}\left(101 \mathrm{MHz}, \mathrm{CDCl}_{3}\right) \delta: 150.6,144.2$, 139.0, 130.7, 129.2, 128.5, 127.4, 127.3, 119.6, 119.4.

氟磺酸 3-(4-甲氧基苯基)苯酚酯(1t): 白色固体, m.p. 52.7 53.7 ${ }^{\circ} \mathrm{C} ;{ }^{1} \mathrm{H}$ NMR $\left(400 \mathrm{MHz}, \mathrm{CDCl}_{3}\right) \delta$ : 7.58 (d, $J=7.5 \mathrm{~Hz}, 1 \mathrm{H}), 7.50(\mathrm{~d}, J=8.8 \mathrm{~Hz}, 4 \mathrm{H}), 7.25$ (d, $J=$ $8.0 \mathrm{~Hz}, 1 \mathrm{H}), 6.99(\mathrm{~d}, J=8.0 \mathrm{~Hz}, 2 \mathrm{H}), 3.85(\mathrm{~s}, 3 \mathrm{sH}) ;{ }^{13} \mathrm{C}$ NMR (101 MHz, $\left.\mathrm{CDCl}_{3}\right) \delta: 160.1,150.6,143.8,130.7$, $128.4,126.9,119.1,118.8,114.6,114.3,55.5$; IR (KBr) $v$ : 3430, 3098, 2967, 1605, 1467, 1446, 1252, 1232, 1142, $932,835,777,682,581 \mathrm{~cm}^{-1}$. HRMS (ESI-TOF) calcd for $\mathrm{C}_{16} \mathrm{H}_{18} \mathrm{FNO}_{3} \mathrm{~S}[\mathrm{M}+\mathrm{H}]^{+}$331.0780, found 331.0758.

氟磺酸 3-(4-叔丁基苯基)苯酚酯(1 u): 白色固体, 75.5 76.5 ${ }^{\circ} \mathrm{C} ;{ }^{1} \mathrm{H}$ NMR (400 MHz, $\left.\mathrm{CDCl}_{3}\right) \delta$ : 7.62 (d, $J=7.6 \mathrm{~Hz}, 1 \mathrm{H}), 7.51(\mathrm{~d}, J=10.0 \mathrm{~Hz}, 6 \mathrm{H}), 7.28$ (d, $J=8.0$ $\mathrm{Hz}, 1 \mathrm{H}), 1.36(\mathrm{~s}, 9 \mathrm{H}) ;{ }^{13} \mathrm{C}$ NMR $\left(101 \mathrm{MHz}, \mathrm{CDCl}_{3}\right) \delta$ : $151.5,150.4,143.9,136.0,130.5,127.0,126.7,126.0$, 119.2, 119.0, 34.6, 31.2; IR (KBr) v: 3438 3085, 2970, 1610, 1451, 1430, 1226, 1138, 932, 833, 693, $587 \mathrm{~cm}^{-1}$. HRMS (ESI-TOF) calcd for $\mathrm{C}_{13} \mathrm{H}_{14} \mathrm{FNO}_{3} \mathrm{~S}[\mathrm{M}+\mathrm{Na}]^{+}$ 283.0440, found 283.0423.

氟磺酸 2-(4-甲氧基苯基)苯酚酯(1v): 无色液体. ${ }^{1} \mathrm{H}$ NMR (400 MHz, $\left.\mathrm{CDCl}_{3}\right) \delta: 7.47(\mathrm{~d}, J=7.9 \mathrm{~Hz}, 3 \mathrm{H}), 7.40$ (d, $J=8.7 \mathrm{~Hz}, 2 \mathrm{H}), 6.97$ (dd, $J=14.2,8.1 \mathrm{~Hz}, 3 \mathrm{H}), 3.83$ (s, $3 \mathrm{H}) ;{ }^{13} \mathrm{C}$ NMR $\left(101 \mathrm{MHz}, \mathrm{CDCl}_{3}\right) \delta: 158.8,133.6,132.1$, 130.4, 129.4, 128.8, 127.8, 55.5; IR (KBr) v: 34512994 , 1757, 1610, 1447, 1246, 1046, 915, 820, $582 \mathrm{~cm}^{-1}$. HRMS (ESI-TOF) calcd for $\mathrm{C}_{13} \mathrm{H}_{11} \mathrm{FNO}_{3} \mathrm{SNa}[\mathrm{M}+\mathrm{Na}]^{+}$ 305.0260 , found 305.0242 .

氟磺酸 2-(4-乙基苯基)苯酚酯(1w): 无色液体. ${ }^{1} \mathrm{H}$ NMR (400 MHz, $\left.\mathrm{CDCl}_{3}\right) \delta: 7.51(\mathrm{~d}, J=6.9 \mathrm{~Hz}, 1 \mathrm{H}), 7.50$ (s, 5H), $7.34(\mathrm{~d}, J=7.5 \mathrm{~Hz}, 2 \mathrm{H}), 3.11 \sim 2.49(\mathrm{~m}, 2 \mathrm{H})$, $1.51 \sim 1.15(\mathrm{~m}, 3 \mathrm{H}) ;{ }^{13} \mathrm{C} \mathrm{NMR}\left(101 \mathrm{MHz}, \mathrm{CDCl}_{3}\right) \delta$ : $147.4,144.6,135.3,132.9$, 132.2, 129.1, 128.9, 128.3, 28.7, 15.5; IR (KBr) v: 3361, 2974, 2892, 1655, 1453, 1383, 1089, 1049, 881, $655 \mathrm{~cm}^{-1}$. HRMS (ESI-TOF) calcd for $\mathrm{C}_{14} \mathrm{H}_{13} \mathrm{FNO}_{3} \mathrm{SNa}[\mathrm{M}+\mathrm{Na}]^{+}$303.0467, found
303.0450 .

氟磺酸 2-(4-叔丁基苯基)苯酚酯(1x): 无色液体. ${ }^{1} \mathrm{H}$ NMR (400 MHz, $\mathrm{CDCl}_{3}$ ) $\delta: 7.53$ (d, J=7.4 Hz, 4H), 7.46 $(\mathrm{s}, 4 \mathrm{H}), 1.41(\mathrm{~s}, 9 \mathrm{H}) ;{ }^{13} \mathrm{C} \mathrm{NMR}\left(101 \mathrm{MHz}, \mathrm{CDCl}_{3}\right) \delta$ : 135.2, 132.6, 132.2, 128.6, 128.9, 125.7, 121.5, 34.8, 31.4; IR (KBr) v: 3358, 2945, 2860, 1682, 1460, 1354, 1025, 1001, 960, $640 \mathrm{~cm}^{-1}$. HRMS (ESI-TOF) calcd for $\mathrm{C}_{16} \mathrm{H}_{17} \mathrm{FNO}_{3} \mathrm{SNa}[\mathrm{M}+\mathrm{Na}]^{+}$331.0780, found 331.0760.

辅助材料(Supporting Information) 联芳环氟磺酸酯 化合物的 ${ }^{1} \mathrm{H}$ NMR 和 ${ }^{13} \mathrm{C}$ NMR 谱图. 这些材料可以免费 从本刊网站(http://sioc-journal.cn/)上下载.

\section{References}

[1] Liu, Z.; Li, J.; Li, S.; Li, G.; Sharpless, K. B.; Wu, P. J. Am. Chem Soc. 2018, 140, 2919.

[2] (a) Revathi, L.; Ravindar, L.; Leng, J.; Rakesh, K. P.; Qin, H.-L. Asian J. Org. Chem. 2018, 7, 662.

(b) Ravindar, L.; Bukhari, S.; Rakesh, K.; Manukumar, H.; Vivek, H.; Mallesha, N.; Xie, Z. Z.; Qin, H. L. Bioorg. Chem. 2018, 81 , 107.

[3] Dong, J.; Krasnova, L.; Finn, M. G.; Sharpless, K. B. Angew Chem., Int. Ed. 2014, 53, 9430.

[4] Veryser, C.; Demaerel, J.; Bieliuunas, V.; Gilles, P.; De Borggraeve, W. M. Org. Lett. 2017, 19, 5244.

[5] Zhou, H.; Mukherjee, P.; Liu, R.; Evrard, E.; Wang, D.; Humphrey, J. M.; Butler, T. W.; Hoth, L. R.; Sperry, J. B.; Sakata, S. K.; Helal, C. J.; Am Ende, C. W. Org. Lett. 2018, 20, 812.

[6] Guo, T.; Meng, G.; Zhan, X.; Yang, Q.; Ma, T.; Xu, L.; Sharpless, K. B.; Dong, J. Angew. Chem., Int. Ed. 2018, 57, 2605.

[7] (a) Biffis, A.; Centomo, P.; Del Zotto, A.; Zecca, M. Chem. Rev. 2018, 118, 2249

(b) Lei, L.; Li, C.; Mo, D. Chin. J. Org. Chem. 2019, 39, 2989 (in Chinese).

(雷禄，李承璟，莫冬亮，有机化学，2019，39，2989.)

[8] (a) Li, H.; Zhao, L.; Liu, Y.; Zhang, X.; Li, W.; Jing, L.; Huang, J.; Wang, W. Chin. J. Org. Chem. 2019, 39, 3207 (in Chinese). (李恒超, 赵玲, 刘燕, 张霞, 李王兵, 敬林海, 黄锦, 汪伟, 有 机化学, 2019, 39, 3207.)

(b) Hooshmand, S. E.; Heidari, B.; Sedghi, R.; Varma, R. S. Green Chem. 2019, 21, 381

[9] (a) Johansson Seechurn, C. C. C.; Kitching, M. O.; Colacot, T. J.; Snieckus, V. Angew. Chem., Int. Ed. 2012, 51, 5062. (b) Suzuki, A. Angew. Chem., Int. Ed. 2011, 50, 6722

[10] (a) Hirakawa, T.; Uramoto, Y.; Mimura, D.; Takeda, A.; Yanagisawa, S.; Ikeda, T.; Inagaki, K.; Morikawa, Y. J. Phys. Chem. B 2017, 121, 164 .

(b) Lennox, A. J. J.; Lloyd-Jones, G. C. Chem. Soc. Rev. 2014, 43 , 412 .

(c) Carrow, B. P.; Hartwig, J. F. J. Am. Chem. Soc. 2011, 133, 2116. (d) Amatore, C.; Duc, G. L.; Jutand, A. Chem.-Eur. J. 2013, 19 , 10082 .

[11] (a) Fu, L.; Cao, X.; Wan, J.;Liu, Y. Chin. J. Chem. 2020, 38, 254. (b) Deraedt, C.; Astruc, D. Acc. Chem. Res. 2014, 47, 494.

[12] (a) Peng, L.; Hu, Z.; Tang, Z.; Jiao, Y.; Xu, X. Chin. Chem. Lett. 2019, 30, 1481.

(b) Xiong, J.; Zhong, G.; Zou, L.; Liu, Y. ChemistrySelect 2018, 3, 8291.

(c) Chen, X.; Hu, C.; Wan, J. P.; Liu, Y. Tetrahedron Lett. 2016, 57, 5116.

(d) Buchspies, J.; Szostak, M. Catalysts 2019, 9, 53 
[13] (a) Campeau, L. C.;Hazari, N. Organometallics 2019, 38, 3. (b) Liu, C.; Li, X. Chem. Rec. 2016, 16, 84.

[14] Liu, C.; Liu, C.; Li, X. M.; Gao, Z. M.; Jin, Z. L. Chin. Chem. Lett. 2016, 5, 631.

[15] Ma, C.; Zhao, C. Q.; Xu, X. T.; Li, Z. M.; Wang, X. Y.; Zhang, K.; Mei, T. S. Org. Lett. 2019, 21, 2464.
[16] Schimler, S. D.; Cismesia, M. A.; Hanley, P. S.; Froese, R. D. J.; Jansma, M. J.; Bland, D. C.;Sanford, M. S. J. Am. Chem. Soc. 2017, $139,1452$.

[17] Li, X.; Feng, F.; Ren, C.; Teng, Y.; Hu, Q.;Yuan, Z. Synlett 2019, $30,2131$.

(Lu, Y.) 